\title{
Pharmacovigilance in Covid-19 vaccines
}

\author{
Hanane Bahouq ${ }^{1,2}{ }^{*}$, Madiha Bahouq $^{3}$, Abdelmajid Soulaymani ${ }^{2}$, and Rachida Soulaymani-Bencheikh ${ }^{4}$ \\ ${ }^{1}$ Hospital of specialties Tangiers, Morocco. \\ ${ }^{2}$ Laboratory of Genetic and Biometry, Faculty of Sciences, Ibn Tofail University, Kenitra, Morocco. \\ ${ }^{3}$ Laboratory of Botany, Biotechnologies and plants Protection, Faculty of Sciences, Ibn Tofail University, Kenitra, Morocco. \\ ${ }^{4}$ Antipoison and Pharmacovigilance Center, Faculty of Medicine and Pharmacy, Mohamed V University, Rabat, Morocco.
}

\begin{abstract}
The ongoing pandemic coronavirus disease of 2019 (COVID-19), originated from Wuhan, China, has caused universal challenging and threatening with considerable health impact and economic losses. Therefore, vaccination, as preventive and protective medical countermeasure, remains an excellent issue for reducing morbidity and mortality of this emerging infectious disease. As of April 2021 and by Emergency Use Authorization (EUA) process, 16 vaccines were authorized by at least one national regulatory authority for public use: two Ribonucleic Acid (RNA) vaccines (Pfizer-BioNTech and Moderna), seven conventional inactivated vaccines (BBIBP-CorV manufactured by Siinopharm, CoronaVac, Covaxin, WIBP-CorV, CoviVac, Minhai-Kangtai and QazVac), five viral vector vaccines (Sputnik Light, Sputnik V, Oxford-AstraZeneca, Convidecia, and Johnson \& Johnson) and two protein subunit vaccines (EpiVacCorona and RBD-Dimer). As other countries, Morocco has established an anti-COVID-19 vaccine strategy in order to effectively contribute to the monitoring of vaccine safety supported by the national platform health vigilance and regulated by the National Anti-Poison and Pharmacovigilance Center (CAPM). In this review, we recorded the main current developed COVID-19 vaccines and discussed pharmacovigilance strategies and tools related safety and tolerability of those therapies. In parallel, a review of the Moroccan experience in this field is also conducted.
\end{abstract}

\section{Introduction}

The ongoing pandemic coronavirus disease of 2019 (COVID-19), originated from Wuhan, China, has caused universal challenging and threatening with considerable health impact and economic losses. The disease is caused by Severe Acute Respiratory Syndrom Coronavirus-2 (SARS-CoV- 2) [1]. To enter into alveolar host cells, the virus uses its Spike proteins by interacting with the Angiotensin Converting Enzyme-2 Receptor (ACE2).

Therefore, the Spike protein is the most targeted for the development of potential COVID-19 vaccines in the virus particles. Actually (as of June 13, 2021), $176,484,738$ persons are infected, 3,812,244 are deaths with fatality rates ranged from 0.24 to $1.49 \%$ [1]. Currently, most of COVID-19 cases are mild or moderate disease with fever, sore throat, cough and dyspnoea but in $5-10 \%$ of cases, disease is severe leading to death.

Due to high risk of transmission, COVID-19 outbreak remains a major challenge for clinicians. Since its emergence, control measures and changes in community's behaviour (such us wearing masks, social isolation and zoning disease) were deployed. In parallel, several agents such as Chloroquine, Hydroxychloroquine, Favipiravir, Monoclonal antibodies, Corticosteroids, Convalescent plasma, Biotherapy (Tocilizumab) and vaccines are being

\footnotetext{
*Corresponding author: hananebahouq@yahoo.fr
}

evaluated. Several clinical trials are ledto identify the most potent remedy or combination against the disease. As a prioritized disease in the research and development (R\&D) blueprint of the WHO, there is an urgent need for effective and safe treatment to contain ongoing outbreaks and to prevent possible ones.

Therefore, vaccination, as preventive and protective medical countermeasure, remains an excellent issue for reducing morbidity and mortality ofthis emerginginfectious disease. Realizing the alarming need for effective-safe vaccines and recognizing the health emergency conditions, Food and Drug Administration (FDA) is facilitating the vaccines rapid availability, developed according to science-based standards for quality, safety and effectiveness [2].

An initiative begun in April 2020 by the WHO, the European Commission and the France government aimed toensure COVID-19 Vaccines Global Access (COVAX).As a part of the Access to COVID-19 Tools ACCELERATOR, COVAX assumes the responsibility of enabling low to middle income countries equitable access to COVID-19 tests, therapies and vaccines [1, 2]. As of April 2021 and by Emergency Use Authorization (EUA) process, 16 vaccines were authorized by at least one national regulatory authority for public use: two Ribonucleic Acid (RNA) vaccines (Pfizer-BioNTech and Moderna), seven conventional inactivated vaccines (BBIBP-CorV 
manufactured

Sinopharm, CoronaVax, Covaxin, WIPBCorV, CoviVac, Minhai-Kangtai and QazVac), five viral vector vaccines (Sputnik Light, Sputnik V, OxfordAstraZeneca, Convidecia, and Johnson \& Johnson) and two protein subunit vaccines (EpiVac Corona and RBDDimer) [2].

Actually, worldwide rapid and massive vaccination coverage, prioritizing elderly and health workers, is tightly assumed to manage COVID-19 disease spread. According to the National Health Ministry, as of April 8, Morocco has received 9.5 million doses of Sinopharm and AstraZeneca COVID-19 vaccines, in addition to the first batch of vaccine delivered under the COVAX program. With one of the fastest vaccination rates in the world, $19,5 \%$ of Moroccan population is fully vaccinated [3]. Despite vaccine's proven efficacy related severe COVID-19 disease prevention, the occurrence of adverse effects has great concerns and had been responsible of vaccine hesitancy [4]. It should be noted that adverse events are unfavourable and unintended signs symptoms or a disease associated with a treatment which must always be recorded on a Case Report Form (CRF). When side effects are studied and established by clinicians, most often resolve on their own, adverse events are completely unpredictable and occur much less often than side effects.

To continuously support the monitoring of COVID19 vaccines safety, a COVID-19 Vaccine Pharmacovigilance Dashboard had been established by the Pan American Health Organisation (PAHO) [5].

As other countries, Morocco has established an antiCOVID-19 vaccine strategy in order to effectively contribute to the monitoring of vaccine safety supported by the national platform health vigilance (https://www.liqahcorona.ma/fr/evenements_indesirables ) and regulated by the National Anti-Poison and Pharmacovigilance Center (CAPM) (https://www.capmsante.ma).

In this review, we recorded the main current developed COVID-19 vaccinesand discussed pharmacovigilance strategies and tools related safety and tolerability of those therapies. In parallel, a review of the Moroccan experience in this field is also reported.

\section{Materials and Methods}

Our searches were collected fromPubMed database, Google Scholar,WHO, FDA, Local Ministries, Health Institutes and Centers for Disease Control and Prevention (CDC), Open Data Government Platform Morocco (https://data.gov.ma/), the National Department for Epidemiology and Disease control (https://www.sante.gov.ma/Publications/Bulletins/Pages) and the National Anti-Poison and Pharmacovigilance Center (CAPM) (https://www.capm-sante.ma).As COVID-19 vaccines technologies and safety monitoring continue to evolve, the information summarized in this review is only current as the date of elaborating this work (June 2021).

\section{Results and discussion}

\footnotetext{
*Corresponding author: hananebahouq@yahoo.fr
}

Results of these literature searches have been included in this following review sections.

Platforms being developed of vaccine are focused on the coronavirus spike protein and its variantsusing "nextgeneration" strategies for precise targeting of the virus mechanisms. They involve nucleic acid technologies, non-replicating viral vectors, peptides, recombinant proteins, live attenuated viruses and inactivated viruses.

We choose to broach the subject through these available types of vaccines.

\subsection{Authorized COVID-19 vaccines}

\subsubsection{RNA vaccines}

The first COVID-19 vaccines to be authorized in the United Kingdom, the United States and the European Union, are the Pfizer-BioNTech COVID-19 vaccine, Moderna COVID-19 vaccine ( December 2020) and the $\mathrm{CVnCoV}$ ( February 2021) [6, 7]. RNA vaccines do not integrateinto host deoxyribonucleic acid (DNA) and use lipid nanoparticle (LNP) delivery system with potential advantages. The LNP system is stabilized by a polyethyleneglycol (PEG) thatprovides a hydrophilic layer, prolonging half-life. They can be manufactured rapidly at low cost with a good safety profile. However, extreme refrigeration to maintain their stability during distribution is required which limits their use in lowincome countries [6]. Due to LNP, RNA vaccines are responsible of allergic reactions including anaphylaxis. PEG, widely used as a stabilizing excipient, is known to be a potential allergen which can induce an IgEmediated reaction and recurrent anaphylaxis $[4,7,8]$.

Therefore, the CDC has recommended the exclusion of any person with a history of a severe or immediate allergic reaction associated with any of the vaccine components [4]. Consequently, a dynamic "safety roadmap" is highly required during vaccination campaign, in order to identify risked populations for severe allergic reactions and to implement prevention strategies [7, 8]. In United States, Vaccine Adverse Event Reporting System (VAERS) is a passive reporting system of early vaccine adverse events co-managed by the CDC and the FDA. Reports to VAERS of adverse events are classified as non-serious or serious (death, life-threatening illness, hospitalization or hospitalization prolongation, persistant disability) adverse event [4].

In addition, a smart phone application is designed to monitor and manage potential long-term safety vaccine problems $[7,8]$.

\subsubsection{Adenovirus vector vaccines (AVVs)}

AAVs are non-replicating viral vector vaccines using a viral vector to deliver genetic material coding for a desired antigen into the host cells. However, replication deficient viruses can be responsible of Vaccine Induced Thrombotic Thrombopenia (VITT) by innate immune responses which activated platelets (platelet factor-4 PF4) and triggered coagulation [9, 10].

As of January 2021, authorized AVVs are the Oxford-AstraZeneca COVID-19 vaccine, the Sputnik V COVID-19 vaccine, Convidecia and the Johnson \& Johnson COVID-19 vaccine. The 
FDA and CDC indicate that AVVs benefits outweighed the risk of developing VITT and hence restarted the EUA Vaccination program [4, 9, 10]. Furthermore, allergic reactions are possible with AVVs, due to the presence of allergenic excipients such as Polysorbate 80 [11].

In our context, the AstraZeneca COVID-19 vaccine is used in vaccination campaign. To date, more than 50\% of the collected adverse events are general benign signs (pain, vaccine injects site swelling, fever, fatigue, headache, muscle pain, nausea, vomiting, itching, chills, muscle pain, arthralgia). Twelve cases of serious allergies have been reported. To manage these allergic incidents, all vaccination sites have been equipped and trained. In addition, four cases of thrombosis (with several thrombogenic factors) have been notified in Morocco. No death case was recorded. The link to the vaccine is still not established (https://www.capmsante.ma).

\subsubsection{Inactivated virus}

Inactivated virus vaccines are using killed virus particles that previously have been grown in culture, but still keeping the surface spike protein intact to stimulate the immune system response [12, 13]. As of January 2021, authorized vaccines of this type are the Chinese CoronaVac, BBIBP-CorV, and WIBP-CorV; the Indian Covaxin; and the Russian CoviVac. Vaccines in clinical trials include the Valneva COVID-19 vaccine [4]. Most of inactivated virus vaccines side effects were mild and moderate. However, four severe adverse events (no detailed in the published article), concluded to be not related to the vaccination were reported with the Sinopharm a whole virus vaccine $[11,13]$.

National pharmacovigilance data do not show an increase in adverse events compared to the rest of the vaccinated population with a notification rate of 2.2 cases per 10,000.Hence, the Adhoc National Scientific Committee for the continual development of the vaccine strategy against Sars-Cov-2 recommendsmaintaining the use of Sinopharm vaccine for the age group of 60 years and over.

\subsubsection{Subunit vaccines}

Subunit vaccines use one or more antigens (often protein subunits) without introducing whole virus particles. As of April 2021, the two authorized Subunit vaccines are the peptide vaccine EpiVac Corona and RBDDimer. The Novavax COVID-19 vaccine, SOBERANA 02 and the Sanofi-GSK vaccine have pending authorizations [4]. The V451 vaccine was terminated due to its misinterpreted potential of HIV testing.

Generally, recombinant protein subunit vaccines are stable and significantly safer than live attenuated and killed vaccines [14]. Also, their production, storage and transportation are less expensive which makes them an adequate and applicable vaccine choice to ensure widespread use, especially in low- and middle-income countries.

Recent scientific researches and laboratory techniques and technologies advances have brought major progress in understanding Human- virus interactions. Virus-like particle vaccines, multiple DNA plasmid vaccines, lentivirus vector vaccines, plant based vaccine, conjugate vaccine and a vesicular stomatitis virus are still in clinical evaluation [14].

Oral vaccines and intranasal vaccines are also being developed.Scientists continue to investigate novel vaccine strategies for prevention of worldwide existing and emerging infectious disease including COVID-19. Furthermore, ongoing monitoring and advanced studies are still required for a better understanding of vaccines mechanisms in order to establish safety evidence by anticipating long-term and delayed rare and serious adverse events.

\subsection{Pharmacovigilance for COVID-19 vaccines strategies}

In fact, the most common pharmacovigilance process is passive one; however, in warning and crisis situations such as, emerging pandemic COVID-19 disease, this approach seems insufficient. An active monitoring pharmacovigilance programs are urgently and constantly required [15].

Nationally, since the emerging of COVID-19 as of March 2020 and under the supervision of Moroccan Health Ministry, the CAPM ensure efficacy diverse therapies and vaccines pharmacovigilance related to this pandemic disease. In 1989, the Center was set up according to the directives of the International Programme on Chemical Safety and included pharmacovigilance and health alert activities (www.capm.ma) and was appointedin 2011, WHO Collaborating Center (WHOCC) for pharmacovigilance [16].

In anticipation of the covid-19 vaccination campaign, the national Health ministry has strengthened information system for reporting side effects via the electronic patient self-Reporting of Adverse-events "Yakada Liqah" application. For every 1000 vaccinations, 1.3 notifications come from both populations and health professionals which demonstrate the effectiveness of the notification system. Then, these notifications are analysed and validated by the CAMP to establish the cause-effect relationship. A weekly national report is also shared with the Health Ministry and the central vaccination committee. It should be noted that adverse events are unfavourable and unintended signs symptoms or a disease associated with a treatment. When side effects are studied and established by clinicians, most often resolve on their own; adverse events are completely unpredictable and occur much less often than side effects.

Generally, the majority of reported adverse events were mild or moderate. Due to COVID-19 specificities patients, the estimation of the event severity remains delicate particularly in intensive care sites. Polymedication and associated co-morbidities are taken into account when analysing the cause-effect relationship using at least two standardized and harmonized imputability methods. Consequently, intensive

\footnotetext{
*Corresponding author: hananebahouq@yahoo.fr
} 
monitoring in vulnerable individuals with increased age and high-risk comorbidities is crucial to manage the potential risk of adverse event [15]. In countries with deficient pharmacovigilance system, the Bayesian network constitutes an option in supporting causality imputation [15].

Besides, notified and validated adverse events are daily notified via the international web-based system "VigiFlow" to be centralized in the international WHO pharmacovigilance VIGIBASE databank [18]. Established since 1978, research and analysis of VIGIBASE are carried out using "Vigilyze" search tool for a global investigating of reported adverse reactions. "VigiFlow" is supporting pharmacovigilance process and vaccine surveillance through the independent Uppsala Monitoring Centre (UMC), as a non-profit foundation for international scientific research, devoted to promote safer medicines [17].

Additionally, awareness campaigns involving health professionals, community partners and social institutes with an effective communication according to the local social and cultural context, must be constantly organized. These measures allow ensuring massive population awareness about the COVID-19 disease and rectify distorted information about vaccines [15].

\section{Conclusion}

An efficient implementation of mass vaccination must be continually accompanied by a multidimensional active pharmacovigilance strategy. The post-vaccination represents a challenge for the pharmacovigilance process [7]. Hence, the vaccine-safety surveillance must passes through a better understanding of adverse events mechanisms.

In urgent context, the rapid vaccine development combined to the uncertain potential long-term adverse events and the emergency authorization for use, motivate rigorous and updated scientific researches related to COVID-19 safety and efficacy. Consequently, strengthening pharmacovigilance strategies remains crucial issue to anticipate, monitor and manage possible vaccines adverse events.

\section{Ethical Approval and Consent to participate}

Not applicable.

\section{Funding}

The authors declare that no funding was received for the present study.

\section{Conflicts of Interest}

The authors declare that they have no conflicts of interest.

\section{References}

1. WHO prioritizing diseases for research and development in emergency contexts, available online from: https://www.who.int/activities/prioritizingdiseases-for-research-and-development-in-emergencycontexts (Accessed 2021 June 13)

2. FDA, COVID-19 vaccines, available online from: https://www.fda.gov/emergency-preparedness-andresponse/coronavirus-disease-2019-covid-19. (Accessed 2021 June 13)

*Corresponding author: hananebahouq@yahoo.fr
3. Coronavirus (COVID-19) Vaccinations: statistics and research. Available online from: https://ourworldindata.org/covid-

vaccinations?country=OWID_WRL. (Accessed 2021 June 14)

4. Centers for Disease Control and Prevention. Possible side effects from vaccines, available online from: https://www.cdc.gov/vaccines/vac-gen/side-effects.htm (Accessed 2021 June 14)

5. COVID-19 Vaccine Pharmacovigilance Dashboard, available online from: https://www.paho.org/en/news/95-2021-covid-19-vaccine-pharmacovigilance-dashboard. (Accessed 2021 June 13)

6. K.S. Park, X. Sun, M.E. Aikins, J.J. Moon, Adv Drug Deliv Rev 169, 137-51 (2021)

7. M.C. Castells, E.J. Phillips, N Engl J Med 384, 643649 (2021)

8. S.A. Meo, I.A. Bukhari, J. Akram, A.S. Meo, D.C. Klonoff, Eur Rev Med Pharmacol Sci 25, 1663-1669 (2021)

9. A. Greinacher, T. Thiele, T.E. Warkentin, K. Weisser, P.A. Kyrle, S. Eichinger, N Engl J Med 384, 2092-2101. (2021)

10. Rare side effects of Adenoviruses vaccines call for careful surveillance, Timmerman Report (April 2021) available online from: https://timmermanreport.com/2021/04/rare-side-effectsof-adenovirus-vaccines-call-for-careful-surveillance/ (Accessed 2021 June 13)

11. N.G. Kounis, I. Koniari, C. de Gregorio, D. Velissaris, K. Petalas, A. Brinia, S.F. Assimakopoulos, C. Gogos, S.N. Kouni, G.N. Kounis, G. Calogiuri, M.Y. Hung, vaccines (Basel) 9, 221 (2021)

12. Medical News Today, Sinopharm COVID-19 vaccine: Should you worry about the side effects?, available online from: https://www.medicalnewstoday.com/articles/sinopharmcovid-19-vaccine-should-you-worry-about-the-sideeffects\#Common-side-effects. (Accessed 2021 June 13) 13. S. Xia, K. Duan, Y. Zhang, D. Zhao, H. Zhang, Z. Xie, X. Li, C. Peng, Y. Zhang, W. Zhang, Y. Yang, W. Chen, X. Gao, W. You, X. Wang, Z. Wang, Z. Shi, Y. Wang, X. Yang, L. Zhang, L. Huang, Q. Wang, J. Lu, Y. Yang, J. Guo, W. Zhou, X. Wan, C. Wu, W. Wang, S. Huang, J. Du, Z. Meng, APan, Z. Yuan, S. Shen, W. Guo, X. Yang, JAMA 324, 951-960 (2020)

14. National Institute of Allergy and Infectious Diseases (NIAID), Vaccines types, available online from: https://www.niaid.nih.gov/research/vaccine-types.

(Accessed 2021 June 13)

15. R. Ferreira-da-Silva, I. Ribeiro-Vaz, M. Morato, J.J. Polónia, Int J Clin Pharm 2, 1-6 (2021)

16. N. Rhalem, R. Aghandous, H. Chaoui, R. Eloufir, N. Badrane, M. Windy, H. Hardouz, L. Ouammi, A. Soulaymani and R. B Soulaymani, Rachida Asia Pac J Med Toxicol 2:82-6 (2013)

17. M. Vogler, H. Ricci Conesa, K. de Araújo Ferreira, F. Moreira Cruz, F. Simioni Gasparotto, K. Fleck, F. 
MacielRebelo, B. Kollross, Y. Silveira Gonçalves?

Pharmaceut Med 34, 327-334 (2020)

*Corresponding author: hananebahouq@yahoo.fr 